\title{
THE ABSORPTION SPECTRA OF THE BLUE SOLUTIONS OF SODIUM AND MAGNESIUM IN LIQUID AMMONIA.
}

By G. E. Gibson And W. L. Argo.

\section{INTRODUCTION.}

THE blue color of solutions of metals in liquid ammonia and alkyl amines has been observed and several of their physical properties have been investigated by Kraus and others. In order to account for the electrical properties of these solutions Kraus ${ }^{1}$ assumes that in all cases the metal dissociates on dissolving according to the equation

$$
\mathrm{Me}=\mathrm{Me}^{+}+\theta \text {, }
$$

where $\mathrm{Me}^{+}$is the cation present in solutions of salts of the metal and $\theta$ is an electron. Both $\mathrm{Me}^{+}$and $\Theta$ may be combined to a greater or less extent with the solvent molecules. From measurements of the electromotive force of concentration cells Kraus ${ }^{2}$ concludes that at least one third of the current in a normal solution of sodium is carried by the unsolvated electron.

The color of these metallic solutions appears to be independent of the metal and the solvent used. They are intensely blue to transmitted light, and, as far as the eye can judge, the shade of color is the same in all cases. In daylight .oor normal solutions of potassium and sodium in ammonia can be matched against ammoniacal copper solutions. This is also true of a saturated solution of magnesium in liquid ammonia.

Similar blue solutions are obtained when sodium is dissolved in molten sodamide ${ }^{1}$ and when calcium is dissolved in a molten mixture of potassium and sodium chloride. ${ }^{3}$

The blue color of these metallic solutions may be due $(a)$ to un-ionized molecules (or atoms) of the metal, $(b)$ to solvated electrons, $(c)$ to unsolvated electrons.

(a) We should expect the color of the un-ionized metal atoms to vary with the metal and to be similar to that of the vapor of the metal, provided the metal atoms were not affected by the presence of the solvent.

1 J. Am. Chem. Soc., 29, r557 (r907).

2 J. Am. Chem. Soc., 36, 864 (I9r4).

${ }^{3}$ Titherley, J. Chem. Soc., 65, 508 (I 894).

${ }^{4}$ A description of this experiment will be published shortly. 
G. E. GIBSON AND W. L. ARGO. [SECOND

Sodium vapor has a purple color. The absorption spectrum of the vapor, however, has been investigated by Wood and others, and is in no way similar to that of the solution in ammonia.

If the un-ionized metal atoms combined with the solvent molecules we should expect the color of the compound to vary with the solvent.

(b) If the color were due to solvated electrons we should expect an absorption band differing from solvent to solvent. It is unlikely that electrons bound to molecules so different in character as ammonia and fused alkali chlorides should produce the same color.

On the whole, therefore, the experimental evidence hitherto obtained favors the assumption $(c)$ that the color is due to unsolvated electrons. In this paper the latter assumption will be tested quantitatively by comparing the theoretical absorption spectrum deduced from this hypothesis with the aid of Drude's theory of metallic dispersion with the actual absorption spectrum determined by experiment.

\section{The Relation between Extinction Coefficient and Wave Length According to Drude's Theory.}

Let us make the same assumptions for the unsolvated electron in the blue solutions that Drude ${ }^{1}$ employs in his electron theory of metallic dispersion and conduction, $i$. e., that the resistance to the motion of the electron through the medium is proportional to its velocity.

The equation of motion of an unsolvated electron is, therefore,

$$
m a=e E-r e^{2} v .
$$

The term on the left is the product of the mass $m$ of the electron and its acceleration $a . \quad E$ is the intensity of the electric field, $e$ the charge on the electron, and $r$ is such a quantity that $r e^{2}$ is the resistance to the motion of an electron moving with unit velocity through the medium. Electrostatic units will be used throughout in this paper.

Equation (I) combined with the equations of the electromagnetic field, leads to the equations

$$
\begin{aligned}
n^{2}\left(\mathrm{I}-\kappa^{2}\right) & =\mathrm{I}+\Sigma \frac{\theta_{h}}{\mathrm{I}-\left(\tau_{h} / \tau\right)^{2}}-4 \pi \frac{m^{\prime} \mathfrak{N}}{r^{2}+\left(m^{\prime} / \tau\right)^{2}}, \\
n^{2} \kappa & =2 \pi \tau \frac{r \Re}{r^{2}+\left(m^{\prime} / \tau\right)^{2}},
\end{aligned}
$$

where $n$ is the refractive index of the solution. The index of absorption $\kappa$ is defined by the equation

$$
J=J_{0} e^{-\frac{4 \pi \kappa}{\lambda} d}
$$

${ }^{1}$ Lehrbuch d. Optik, Leipzig, I9I2, p. 386. 
where $J_{0}$ is the intensity of the incident ray of wave-length $\lambda$ and $J$ is the intensity after a layer of thickness $d$ has been traversed. The symbol $m^{\prime}$ is the ratio of the mass of the electron to the square of its charge $\left(m^{\prime}=m / e^{2}\right) . \quad \mathfrak{N}$ is the number of unsolvated electrons in a cubic centimeter of the solution. The radian time $\tau$ is equal to the periodic time divided by $2 \pi$. $\theta_{h}$ and $\tau_{h}$ are constants of the bound electrons.

Equations (2) are the general equations of Drude for an absorbing medium containing both bound and unbound electrons. The term under the sign of summation in the first of equations (2) represents the influence of the bound electrons.

For a colorless solvent both $\kappa$ and $\tau_{h} / \tau$ are very small in the visible spectrum. The refractive index of the pure solvent, therefore, varies very slightly with the wave-length, and will be assumed to be constant in the following. In terms of the symbols of equations (2) its value would be given by

$$
n_{0}^{2}=\mathrm{I}+\Sigma \theta_{h} .^{1}
$$

Neglecting $\tau_{h} / \tau$ in equations (2) and eliminating $n$ we obtain

$$
\frac{\mathrm{I}-\kappa^{2}}{\kappa}=\left(\frac{n_{0}^{2} r}{2 \pi \mathfrak{l}}-\frac{2 m^{\prime}}{r}\right) \frac{\mathrm{I}}{\tau}+\frac{n_{0}^{2} m^{\prime 2}}{2 \pi \mathfrak{N} r} \cdot \frac{\mathrm{I}}{\tau^{3}} .
$$

In terms of the wave-length $\lambda=2 \pi c \tau$ and the extinction coefficient $\epsilon=\left(4 \pi \kappa \log _{10} e\right) / \lambda$ this becomes

$$
\frac{a^{2}}{\epsilon}-\epsilon \lambda^{2}=2 \pi a c\left(\frac{n_{0}^{2} r}{2 \pi \Re}-\frac{2 m^{\prime}}{r}\right)+\frac{4 \pi^{2} a c^{3} n_{0}^{2} m^{\prime 2}}{\mathfrak{N} r} \cdot \frac{\mathbf{I}}{\lambda^{2}},
$$

where $a$ is a contraction for $4 \pi \log _{10} e$.

Except for very large values of $\epsilon$ (such as occur in pure metals) the term $\epsilon \lambda^{2}$ may be neglected in the visible spectrum, and (5a) may therefore be written

$$
\frac{a^{2}}{\epsilon}=b_{1}+\frac{b_{2}}{\lambda^{2}}
$$

where $b_{1}$ and $b_{2}$ are independent of the wave-length, $i . e$. ,

$$
\begin{aligned}
& b_{1}=2 \pi a c\left(\frac{n_{0}^{2} r}{2 \pi \Re}-\frac{2 m^{\prime}}{r}\right), \\
& b_{2}=\frac{4 \pi^{2} a c^{3} n_{0}^{2} m^{\prime 2}}{\mathfrak{N} r} .
\end{aligned}
$$

Equation (6) expresses the theoretical relationship between the extinction

1 The refractive index of liquid ammonia $n_{0}$ has not been determined. In the calculations $n_{0}{ }^{2}$ was taken to be equal to 2 as the refractive index of liquid ammonia cannot be far from that of water. 
coefficient $\epsilon$ and the wave-length $\lambda$ in the visible spectrum. According to (6) $a^{2} / \epsilon$ is a linear function of $\mathrm{I} / \lambda^{2}$.

If $2 m^{\prime} / r$ is negligible compared with $\left(n_{0}{ }^{2} r\right) / 2 \pi \Re$ and if $\lambda$ is small enough to make $\epsilon \lambda^{2}$ negligible compared with $a^{2} / \epsilon$ the extinction coefficient $\epsilon$ for a given wave length will be proportional to the concentration $\mathfrak{R}$ of the unsolvated electrons. These are therefore necessary conditions that Beer's law shall hold.

For sufficiently large wave-lengths we see from $(5 a)$ that

$$
\frac{a^{2}}{\epsilon}-\epsilon \lambda^{2}=0,
$$

or

$$
\epsilon=\frac{a}{\lambda} .
$$

In other words $\epsilon$ is independent of the concentration when $\lambda$ is large. Hence Beer's law must cease to hold when the wave-length exceeds a certain amount determined by the values of $b_{1}$ and $b_{2}$. The extinction coefficient vanishes for infinite wave-lengths in all cases.

For very short wave-lengths $\left(5^{a}\right)$ reduces to

$$
\frac{a^{2}}{\epsilon}=\frac{4 \pi^{2} a c^{3} m^{\prime 2} n_{0}^{2}}{r \Re \mathfrak{i}} \cdot \frac{\mathrm{I}}{\lambda^{2}},
$$

so that in this case $\left(b_{1}=0\right)$ Beer's law is always obeyed.

If $b_{1}$ is negative we must have $2 m^{\prime} / r>\left(n_{0}{ }^{2} r\right) / 2 \pi \Re$ and Beer's law cannot hold, since $b_{1}$ is no longer inversely proportional to $\mathfrak{A}$.

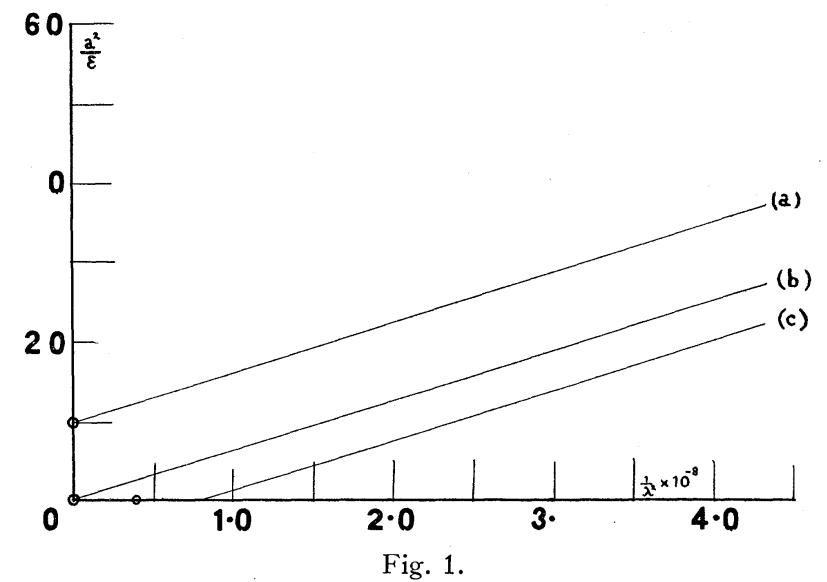

The graphs of $a^{2} / \epsilon$ against $\mathbf{I} / \lambda^{2}$ as they follow from the general equation $(5 a)$ are drawn in Fig. I. There are three types of curve, $a, b$, and $c$, according as $b_{1}$ is positive, zero, or negative. The equation of the asymp- 
tote in all cases is equation (6) viz.,

$$
\frac{a^{2}}{\epsilon}=b_{1}+\frac{b_{2}}{\lambda^{2}} .
$$

On the scale of Fig. I the remainders of the three curves $a, b, c$ are indistinguishable from the axes. The minima of $a^{2} / \epsilon$ are marked with circles.

A more elaborate theory of metallic dispersion has recently been developed by Jaffé ${ }^{1}$ in which the frictional resistance $r$ is assumed to be due to collisions between the free electrons and the atoms of the medium. In place of Drude's equations (2) his theory leads in our case to the equations

$$
\begin{aligned}
n^{2}\left(\mathrm{I}-\kappa^{2}\right) & =n_{0}{ }^{2}+\frac{2 \sqrt{\pi^{3} \sigma}}{\nu} \psi_{1}, \\
n^{2} \kappa & =\frac{2 \pi \sigma}{\nu} \psi_{2},
\end{aligned}
$$

where $\psi_{1}$ and $\psi_{2}$ are functions of the argument $z=(3 \sqrt{ } \pi / 4)\left(m^{\prime} / r\right) \nu$. For large values of $z$ we obtain from Jaffé's theory

$$
\begin{aligned}
& \psi_{1}=-\frac{3}{2} \cdot \frac{\mathrm{I}}{z} \\
& \psi_{2}=\frac{2}{z^{2}} .
\end{aligned}
$$

Substituting these values of $\psi_{1}$ and $\psi_{2}$ in (8), and eliminating $n$, we obtain in place of equation $(5 a)$

$$
\frac{a^{2}}{\epsilon}=-\frac{9 \pi^{2}}{8} \frac{a m^{\prime} c}{r}+\frac{9 \pi^{3}}{8} \frac{n_{0}^{2} a m^{\prime 2} c^{3}}{\mathfrak{N} r} \cdot \frac{\mathrm{I}}{\lambda^{2}} .
$$

When $\left(n_{0}^{2} r\right) / 2 \pi r$ is negligible this equation is identical in form with $\left(5^{a}\right)$ and differs only slightly in the values of the constants. Jaffé's theory therefore leads to the same conclusions as the theory of Drude.

\section{The Theories of Drude and Jaffé in view of the Experi-} mental Determinations.

With a view to testing the hypothesis that the blue color is due to unsolvated electrons, the absorption spectra of solutions of sodium and magnesium in liquid ammonia were determined in the visible spectrum. The results of these experiments will be discussed now and compared with the requirements of the theories of Drude and Jaffé while the details of the experiments will be found in Section IV.

${ }^{1}$ Ann. d. Physik [4], 45, 1217, I914. 
In Fig. 2 the values of $a^{2} / \epsilon$ calculated from our determinations (See col. IX. and X., Table II., and col. XI. and XIII., Table VII.) are plotted against $\mathrm{I} / \mathrm{\lambda}^{2}$. There are three curves for sodium at different concentrations, $\mathrm{Na}(\mathrm{I}), \mathrm{Na}(2)$ and $\mathrm{Na}(3)$ (circles), and one for a nearly saturated solution of magnesium (triangles).

The concentration of sodium is $2.8 \times \mathrm{IO}^{-3}$ normal in series (I) and

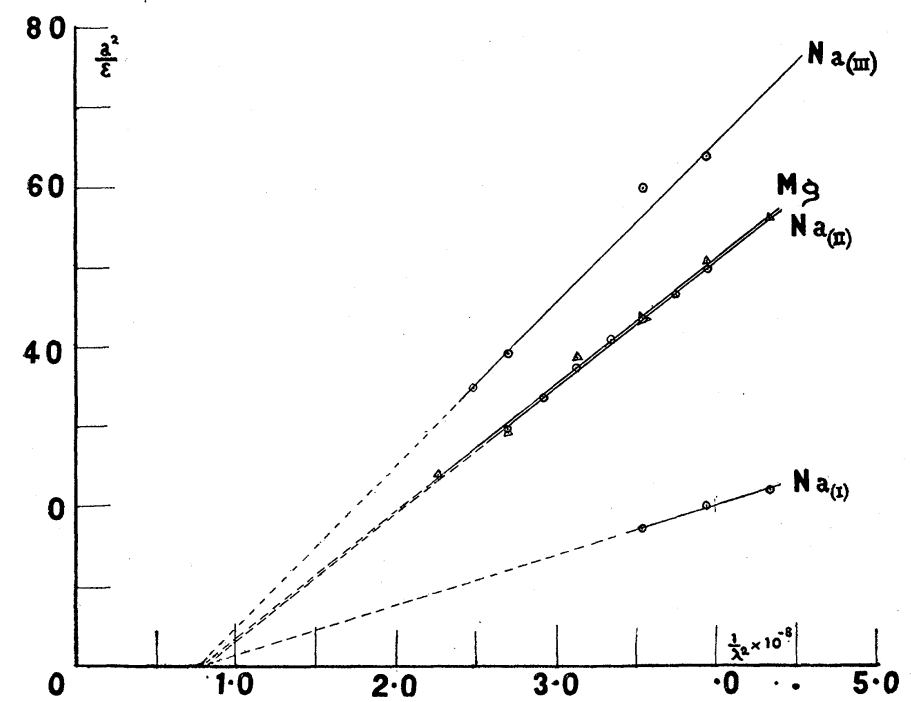

Fig. 2.

I. $3 \times \mathrm{IO}^{-3}$ normal in series (2). The concentration in series (3) is less chan $\mathrm{I} .3 \times \mathrm{IO}^{-3}$ normal, but was not determined exactly. No attempt was made to determine the concentration of the magnesium solution.

The curve for magnesium (Fig. 2) coincides, within the limits of experimental error, with one of the sodium curves. This is strong evidence in favor of the assumption that the coloring principle is the same for both metals.

Within the limits of error the graphs are all straight lines and intersect at a common point on the axis of abscissæ. In other words, the ratio of the ordinates of the various curves at a given wave-length is independent of the wave-length. While this does not prove that Beer's law is obeyed, it is one of the necessary conditions for the proportionality of the extinction coefficient and the concentration of the coloring molecules.

If Beer's law were true for the total dissolved sodium, the concentration $N$ of sodium multiplied by the value of $a^{2} / \epsilon$ should be constant for all wave-lengths. This is approximately confirmed by our experiments. At wave-length $\lambda=502 \mu \mu$ we have $\left(a^{2} / \epsilon\right) N=0.56$ for series (I) and 
$\left(a^{2} / \epsilon\right) N=0.65$ for series (2). The difference between these two values can be accounted for by the loss of sodium due to the amide reaction in the time which elapsed between the two measurements (see Section IV., $d)$. Beer's law therefore holds approximately for the total dissolved sodium.

The equation ( 5 a) obtained from Drude's theory in Section II. may be tested now in the light of these measurements.

The values of $b_{1}$ and $b_{2}$ obtained by the method of least squares from the experimental data are given in columns II. and III. of Table I.

TABLE I.

\begin{tabular}{|c|c|c|c|c|c|c|c|c|}
\hline $\begin{array}{c}\text { I. } \\
\text { Series. }\end{array}$ & $\begin{array}{c}\text { II. } \\
b_{1}(\text { Exp.). }\end{array}$ & $\begin{array}{c}\text { III. } \\
b_{2} \times 10^{8} \\
(\mathbf{E x p} .) .\end{array}$ & $\begin{array}{c}\text { IV. } \\
\mathfrak{n} \times \mathrm{ro}^{-20} \\
(\mathrm{Calc} .) .\end{array}$ & $\begin{array}{c}\mathrm{v} . \\
r \\
\text { (Calc.). }\end{array}$ & $\begin{array}{c}\text { VI } \\
N \text { (Mols } \\
\text { per Liter) } \\
\text { (Calc.). } \\
\end{array}$ & $\begin{array}{c}\text { VII. } \\
N(\text { Exp.) } \\
\text { (Mols.per } \\
\text { Liter). }\end{array}$ & $\begin{array}{c}\text { VIII. } \\
K \times \text { I0 }^{-5} \\
\text { (Calc.). }\end{array}$ & $\begin{array}{c}\text { IX. } \\
K \times \text { Io }^{3} \\
(\text { Kraus }) . \\
\end{array}$ \\
\hline $\mathrm{Na}(1)$ & -4.57 & 6.18 & 1.672 & 1801 & 0.271 & 0.00281 & 1.03 & 2.92 \\
\hline $\mathrm{Na}(2)$ & -12.83 & 15.97 & 1.715 & 641 & 0.295 & 0.00130 & 3.14 & 1.35 \\
\hline $\mathrm{Na}(3)$ & -15.38 & 20.28 & 1.816 & 535 & 0.278 & & 3.56 & \\
\hline $\mathrm{Mg}$ & -12.21 & 15.88 & 1.738 & 674 & 0.281 & & 2.86 & \\
\hline
\end{tabular}

Columns IV. and V. contain the values of the number of electrons $\mathfrak{R}$ per cubic centimeter and the frictional resistance constant $r$ as they would follow from Drude's theory. Column VI. contains the concentration of free electrons in mols per liter calculated from the values of $\Re$ in column IV. Column VII. contains the concentration of sodium determined by experiment. Column VIII. contains the values of the conductivity $K\left(\mathrm{ohm}^{-1} \mathrm{~cm} \cdot{ }^{-1}\right)$ calculated from the values of $\mathfrak{N}$ and $r$ in columns IV. and V., and column IX. the specific conductivity derived from the experiments of Kraus. ${ }^{1}$

Drude's theory, therefore, gives values for the conductivity which are about thirty million times too large. Thus the specific conductivity $K$ of a .0028 normal solution of sodium is $2.92 \times 1 \mathrm{IO}^{-3} \omega^{-1} \mathrm{~cm} .{ }^{-1}$ while the conductivity calculated from Drude's theory for the same solution is 1.03 $\times \mathrm{Io}^{5} \omega^{-1} \mathrm{~cm} .^{-1}$ (see Table I.).

Drude's theory also leads us to conclude that Beer's law would not be even approximately true for the free electrons. The calculated concentrations of free electron are very great and independent of the concentration of sodium. For each sodium atom in a .oor normal solution Drude's theory would require about one thousand electrons, and we should have to ascribe the increased depth of color on adding more sodium to an increased frictional resistance to the motion of the electrons without any increase in their number.

${ }^{1}$ J. Am. Chem. Soc., 36, 877 (I9I4). 
If, therefore, we grant the validity of Drude's theory we are forced to conclude that the blue color of these solutions is not wholly due to unsolvated electrons.

It is important to determine the effect on the coloring principle of changing the metal and the solvent. With this end in view we propose to continue our measurements with other metals such as potassium and lithium and with other solvents such as methylamine, ethylamine, hydrazine and possibly with fused salts.

\section{IV (a). The Spectrophotometer.}

The absorption spectrum was measured by means of a spectrophotometer of the type described by Grünbaum and Martens. ${ }^{1}$ A source of light $A$ (Fig. 3) illuminates the ground-glass window $B$. The light from

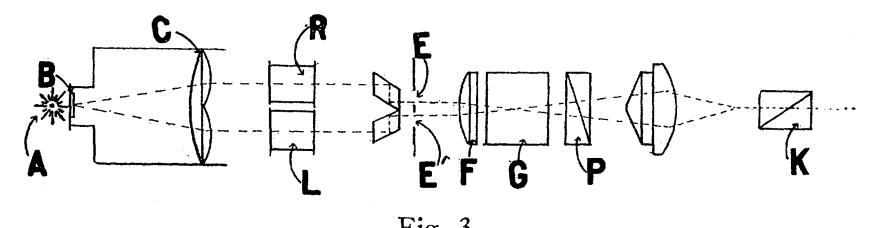

Fig. 3.

$B$ passes through two lenses $C$, which produce an image of $B$ on the slits $E, E^{\prime}$. The two beams pass respectively through a cell $R$ containing the solution to be examined, and a cell $L$ containing water which is used to compensate for the loss of light due to reflection from the windows of the cell. After passing through the slits $E, E^{\prime}$ the two beams traverse successively the collimator $F$, the prism $G$, the polarizer $P$ and the analyzer $K$. The analyzer is rotated until the fields illuminated by the two beams appear of equal intensity.

The calculation of the extinction coefficient from the angle of rotation is discussed in section IV $(d)$.

The source of illumination is an acetylene flame, one inch wide and shielded by a metal chimney three inches in diameter and about two feet high. The flame, when so arranged, burns steadily and illuminates the ground glass more uniformly than a tungsten filament lamp.

The arbitrary wave-length scale of the spectrophotometer had already been calibrated by Adams and Rosenstein ${ }^{2}$ in this laboratory. The calibration was repeated for the sodium $D$ lines and agreed exactly with their determination.

${ }^{1}$ Ann. der Phys., I2, 984 (r903).

${ }^{2}$ J. Am. Chem. Soc., 36, I452 (r9I4). 
IV (b). The Absorption Cell.

The ordinary metal absorption cell with plane glass windows cannot be used for solutions of metals in liquid ammonia. In the first instance, the cell must be perfectly airtight, as the least trace of moisture is sufficient to destroy the blue color. It must also be capable of withstanding a pressure of eight to ten atmospheres, which is the vapor pressure of liquid ammonia at room temperatures. The first cells used were made of copper with glass windows pressed tightly against a thin flange of the metal. Although it was possible to obtain a vacuum-tight joint in this way, the cells were difficult to clean, and slight traces of impurity, such as solder or brass, catalyzed the amide reaction and made it difficult to obtain a permanent blue color.

The cell finally adopted was made entirely of quartz. It is shown in completed form in Fig. 4. The two plates $A, A$, one centimeter in diameter, were made of optical quartz free from bubbles. They were

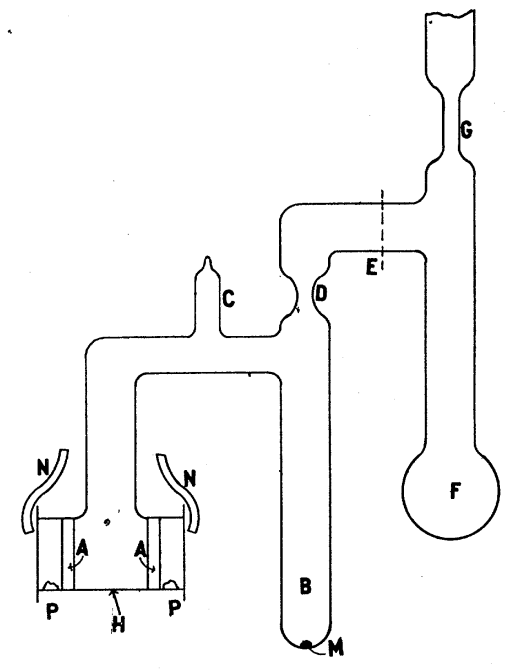

Fig. 4.

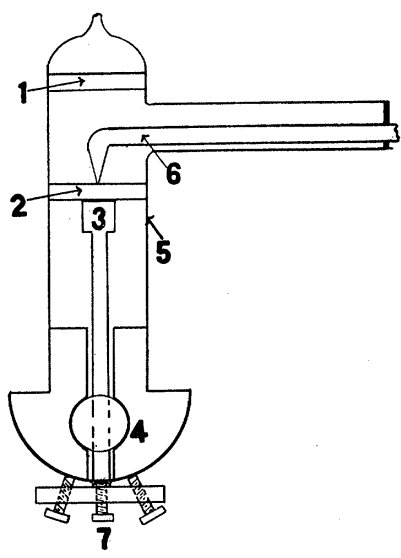

Fig. 5.

ground plane parallel and were fused into the quartz tube $H$, by means of the oxygen gas flame, in the following manner:

The window I, Fig. 5, was first sealed into the quartz test tube 5 in a position as nearly perpendicular to the axis as possible. The window 2 was then adjusted parallel to I by means of the appliances shown. A cylindrical block of quartz 3 was ground plane on its upper surface and connected by a quartz rod with the universal joint 4. By manipulating the three screws, 7 , the upper surface of the block could be adjusted to a position making any desired angle with the axis of the tube. The window 
2 was kept in close contact with the block, 3 , by means of a quartz pointer, 6 , which was depressed by a glass spring not shown in the figure. The window 2 was then adjusted until the image of a point source of light reflected from its surface coincided exactly with the image of the same source reflected from the window, I. The source, which consisted of a small loop of incandescent platinum wire, was placed in the line of the normal to the two windows at a distance of about $150 \mathrm{~cm}$. from the cell. The reflected images were observed through the incandescent loop. The two windows could be adjusted in this way to within Io $^{\prime}$ of arc. Window 2 was then sealed into position by means of the oxygen gas flame. ${ }^{1}$

The two ends of the quartz tube were then cut off, so as to leave an edge of about $3 \mathrm{~mm}$. beyond the windows. The completed cell is shown in Fig. 4.

\section{IV $(c)$. The Method of Filling the Cell.}

The system $A B C D$ in Fig. 4 was made entirely of quartz. Beyond the Khotinsky cement seal $E$ glass was used throughout. The whole system was first evacuated and left in contact with phosphorus pentoxide for several hours. After the apparatus had been washed out several times with dry ammonia gas a quantity of ammonia sufficient to fill the cell was distilled into the bulb $F$ by immersing it in alcohol cooled to $-60^{\circ} \mathrm{C}$. The bulb $F$ contained a small piece of sodium which dissolved in the ammonia and removed the last traces of moisture. The constriction $G$ was then sealed and the ammonia in $F$ distilled into the tube $B$, which contained a portion $M$ of the metal to be examined. After the cell had been sealed off at the constriction $D$ by means of the oxygen-gas flame it was ready for the measurements.

IV $(d)$. Measurements with Sodium in Liquid Ammonia at $-33.5^{\circ} \mathrm{C}$.

The quartz cell was surrounded with a copper jacket $A$ (Fig. 6), which could be filled with boiling liquid ammonia through the tube $B$. The ammonia vapor was led out through the tube $C$ to a large flask filled with water where it was absorbed. Copper filings were placed between the quartz cell and the copper jacket in order to secure good conduction of heat. The jacket was then fitted into a wooden cylinder $D$ which served the double purpose of a heat insulator and a support for the cell in the spectrophotometer.

To prevent the deposition of moisture on the cold quartz windows,

${ }^{1}$ It is difficult to avoid entirely the sublimation of silica on the windows in the process of sealing them into the tube. The slight irregularities resulting from this became invisible on the inner surfaces when the cell was filled with liquid ammonia. The fog on the outside of the windows was made to disappear by cementing thin cover glasses to them with Canada balsam. 
cover glasses were cemented with Khotinsky cement to the projecting edges of the cell, in the manner shown in Fig. 4. The air chambers were dried by small pellets of partly deliquesced phosphorus pentoxide, $p, p$. Jets of dry air were directed against the outer surfaces of the cover glasses by means of the tubes $N, N$. When the air jets were adjusted correctly they prevented entirely the deposition of moisture.

After the cell had been dried by evacuation, pieces of capillary tube of known length and diameter, full of sodium, were introduced through $C$ (Fig. 4). The cell was then filled as described in paragraph IV $(c)$. The amount of sodium introduced into the cell was more than sufficient for the highest concentration desired. After mixing thoroughly the position of the meniscus of the solution in the tube $B$ was measured. A portion of the solution was then transferred to the cell $H$, and the position of the meniscus in $B$ was again measured. By cooling the cell the remainder of the solvent was

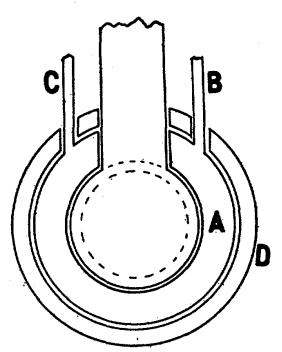

Fig. 6. distilled from $B$ to $H$. This process was repeated until the desired dilution was obtained. The volumes corresponding to the various positions of the meniscus in $B$ were determined after the tube had been opened at the end of the experiment.

The method of reading the spectrophotometer and the method of averaging the readings were essentially the same as those of Grünbaum and Martens. ${ }^{1}$ Grünbaum and Martens compare the cell containing the colored solution with a cell of the same dimensions containing the pure solvent. This method would have necessitated the construction of a second quartz cell, and a duplication of the cooling apparatus. To avoid this, the colored solution and the pure solvent were compared in the same cell at different times with a glass cell containing water. The water cell was used to compensate approximately for the reflections from the windows of the quartz cell. Under these conditions the sensibility of the readings is improved.

A complete determination at any wave-length involves the determination of four angles:

I. The angle $\alpha_{1}$, with the quartz cell containing liquid ammonia in position $R$ (Fig. 3) and the glass water cell in position $L$.

2. The angle $\alpha_{2}$, with the quartz cell containing liquid ammonia in position $L$ and the glass water cell in position $R$.

3. The angle $\alpha_{3}$, with the quartz cell containing sodium solution in position $R$ and the glass water cell in position $L$.

1 Loc. cit. 
4. The angle $\alpha_{4}$, with the quartz cell containing sodium solution in position $L$ and the glass water cell in position $R$.

The glass water cell might be replaced by an imaginary quartz cell excactly similar to the cell containing the ammonia solution but filled with a hypothetical liquid of extinction coefficient such that the fraction of the incident light absorbed by the hypothetical cell would be exactly equal to the fraction absorbed by the actual glass water cell. The difference between the extinction coefficient $\epsilon_{\mathrm{NH}_{3}}$ of the pure liquid ammonia and the hypothetical extinction coefficient $\epsilon_{h}$ is then given by the relation

$$
\epsilon_{\mathrm{NH}_{3}}-\epsilon_{h}=\frac{\log \tan \alpha_{2}-\log \tan \alpha_{1}}{d},
$$

where $d$ is the length of the column of liquid traversed by the light in the quartz cell.

The difference between the extinction coefficient $\epsilon_{\mathrm{Na}}$ of the blue solution and $\epsilon_{h}$ is

$$
\epsilon_{\mathrm{Na}}-\epsilon_{h}=\frac{\log \tan \alpha_{4}-\log \tan \alpha_{3}}{d} .
$$

Hence the required difference between the extinction coefficients of the blue solution and the pure solvent (liquid ammonia) is

$$
\epsilon_{\mathrm{Na}}-\epsilon_{\mathrm{NH}_{3}}=\frac{\log \tan \alpha_{4}-\log \tan \alpha_{3}-\log \tan \alpha_{2}+\log \tan \alpha_{1}}{d} .
$$

Even at $-33.5^{\circ} \mathrm{C}$. the fading of the blue color due to the amide reaction is sufficiently rapid to necessitate correction. The angles $\alpha_{3}$ and $\alpha_{4}$ for each wave-length were therefore determined at measured intervals and plotted against the time of reading. The values of $\alpha_{3}$ and $\alpha_{4}$ at the time of the first reading of the series were obtained by graphical extrapolation. The largest correction applied to any reading amounted approximately to 4 per cent. of the extinction coefficient. The error in the graphical extrapolation is certainly not greater than 25 per cent. of this correction, so that the resultant error in the extinction coefficient due to this cause cannot exceed I per cent. This maximum error applies only to reading (6), series III., in the table below. The uncertainty in the fading correction is much less for all other readings (see Column VIII., Table II.).

\section{IV (e). Measurements with Magnesium.}

Cottrell ${ }^{1}$ had observed that solutions of magnesium in ammonia were extraordinarily stable even at room temperature. Magnesium was

${ }^{1}$ J. Phys. Chem., I8, 85 (I9r4). 
TABLE II.

Temperature $=-33 \cdot 5^{\circ} \mathrm{C} . \quad$ Length of Column $d=1.523 \mathrm{~cm}$.

\begin{tabular}{|c|c|c|c|c|c|c|c|c|c|c|c|c|}
\hline & \multirow{2}{*}{ 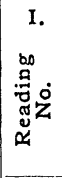 } & \multirow{2}{*}{ 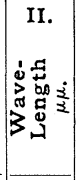 } & \multirow{2}{*}{ 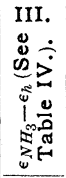 } & \multirow{2}{*}{ 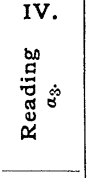 } & \multirow{2}{*}{ 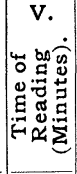 } & \multirow{2}{*}{ 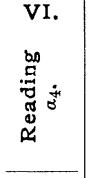 } & \multirow{2}{*}{ 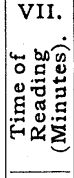 } & \multicolumn{2}{|c|}{$\begin{array}{l}\text { VIII. } \\
\text { Corrected } \\
\text { Reading. }\end{array}$} & \multirow{2}{*}{ 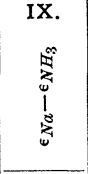 } & \multirow{2}{*}{$\begin{array}{l}\mathrm{X} . \\
\frac{a^{2}}{\epsilon}\end{array}$} & \multirow{2}{*}{ 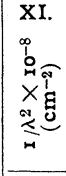 } \\
\hline & & & & & & & & $\alpha_{3}$ & $a_{4}$ & & & \\
\hline Series (1). . & (1) & 529 & .116 & $87.09^{\circ}$ & 0 & $1.87^{\circ}$ & 6 & $87.1^{\circ}$ & $1.75^{\circ}$ & 1.73 & 17.2 & 3.54 \\
\hline Conc. of $\mathrm{Na}=$ & (2) & 502 & .123 & 85.13 & 14 & 2.81 & 18 & 85.4 & 2.5 & 1.48 & 20.0 & 3.94 \\
\hline $2.8 \times 10^{-6} \mathrm{mols}$ & (3) & 480 & .133 & 83.23 & 36 & 3.58 & 30 & 84.0 & 3.0 & 1.35 & 22.0 & 4.33 \\
\hline per cc. & (4) & 529 & & 86.14 & 44 & 2.58 & 47 & & & & & \\
\hline Series $(2) \ldots \ldots$ & (1) & 608 & .098 & 79.48 & 0 & 6.84 & 3 & 79.7 & 6.7 & 0.99 & 29.8 & 2.70 \\
\hline Conc. of $\mathrm{Na}=$ & (2) & 585 & .107 & 76.88 & 8 & 8.32 & 5 & 77.4 & 8.0 & .880 & 33.8 & 2.92 \\
\hline $1.3 \times 10^{-6} \mathrm{mols}$ & (3) & 564 & .112 & 74.62 & 9 & 9.69 & 11 & 75.3 & 9.0 & .745 & 37.5 & 3.13 \\
\hline \multirow[t]{13}{*}{ per cc. } & (4) & 546 & .114 & 72.66 & 15 & 10.87 & 13 & 73.7 & 10.1 & .728 & 40.9 & 3.34 \\
\hline & (5) & 529 & .116 & 70.76 & 18 & 12.00 & 20 & 72.0 & 10.6 & .682 & 43.6 & 3.54 \\
\hline & (6) & 515 & .119 & 69.38 & 25 & 12.87 & 22 & 71.0 & 11.6 & .636 & 46.7 & 3.74 \\
\hline & (7) & 502 & .123 & 68.32 & 27 & 14.00 & 28 & 70.1 & 12.5 & .596 & 49.9 & 3.94 \\
\hline & (8) & 608 & & 77.54 & 34 & 7.93 & 30 & & & & & \\
\hline & (9) & 608 & & 77.37 & 41 & 8.08 & 44 & & & & & \\
\hline & $(10)$ & 529 & & 69.17 & 48 & 12.99 & 46 & & & & & \\
\hline & (11) & 608 & & 76.74 & 50 & 9.30 & 53 & & & & & \\
\hline & $(12)$ & 529 & & 68.34 & 58 & 13.34 & 56 & & & & & \\
\hline & (13) & 608 & & 75.22 & 64 & 9.09 & 67 & & & & & \\
\hline & (14) & 529 & & 67.04 & 72 & 13.92 & 69 & & & & & \\
\hline & (15) & 608 & & 73.84 & 88 & 11.92 & 91 & & & & & \\
\hline & $(16)$ & 529 & & 65.75 & 98 & 15.63 & 94 & & & & & \\
\hline \multirow[t]{18}{*}{$\operatorname{Series}_{-}{ }^{-}(3) \ldots \ldots$} & (1) & 608 & .098 & 74.31 & 0 & 10.18 & 3 & 74.3 & 10.2 & .753 & 39.5 & 2.70 \\
\hline & (2) & 635 & .084 & 75.60 & 13 & 9.34 & 7 & 76.6 & 8.7 & .860 & 34.9 & 2.48 \\
\hline & (3) & 608 & .098 & 72.83 & 16 & 11.45 & 18 & & & & & \\
\hline & (4) & 529 & .116 & 65.13 & 25 & 16.09 & 22 & 66.6 & 15.1 & .496 & 60.0 & 3.54 \\
\hline & (5) & 502 & .123 & 63.25 & 32 & 17.09 & 39 & 65.0 & 15.3 & .464 & 64.1 & 3.94 \\
\hline & (6) & 480 & .133 & 59.28 & 59 & 18.13 & 48 & 62.0 & 15.7 & .409 & 72.8 & 4.33 \\
\hline & (7) & 608 & & 68.98 & 63 & 13.88 & 68 & & & & & \\
\hline & (8) & 608 & & 65.90 & 102 & 15.96 & 99 & & & & & \\
\hline & (9) & 564 & & 62.24 & 106 & 18.65 & 109 & & & & & \\
\hline & $(10)$ & 529 & & 59.64 & 117 & 20.59 & 113 & & & & & \\
\hline & $(11)$ & 502 & & 58.20 & 121 & 20.71 & 127 & & & & & \\
\hline & $(12)$ & 480 & & 55.31 & 140 & 21.08 & 132 & & & & & \\
\hline & (13) & 608 & & 62.07 & 150 & 19.33 & 154 & & & & & \\
\hline & (14) & 564 & & 58.68 & 159 & 21.35 & 158 & & & & & \\
\hline & $(15)$ & 529 & & 57.00 & 162 & 22.44 & 163 & & & & & \\
\hline & $(16)$ & 502 & & 55.70 & 168 & 23.03 & 165 & & & & & \\
\hline & $(17)$ & 491 & & 54.55 & 171 & 23.52 & 177 & & & & & \\
\hline & (18) & 608 & & 59.70 & 183 & 20.85 & 181 & & & & & \\
\hline
\end{tabular}


therefore chosen for our earlier measurements in order to avoid the difficulties involved in cooling the cell. The vapor pressure of liquid ammonia is about eight atmospheres at room temperature, and two cells exploded under this pressure before a measurement was obtained. The strength of the cell was then increased by diminishing its size considerably, but the apparatus with which the determination was finally obtained exploded just as the series of measurements had been completed. The cell, however, was intact, and the explosion may have been caused by the pressure of a metal clamp which was used to mount the apparatus in the spectrophotometer.

As the construction of the quartz cells is somewhat troublesome, our subsequent experiments with sodium were performed at the boiling point of ammonia as described in paragraph IV $(d)$, in order to avoid the risk of explosion.

Except that the cell was not cooled, the method of performing the measurements was the same for magnesium as for sodium. The magnesium was introduced in the form of freshly cut turnings. It was found necessary to leave the magnesiuin in contact with the liquid ammonia for at least a week before the blue color appeared. This delay in the appearance of the color is probably due to the formation of a protecting layer of hydroxide on the surface of the metal.

The fading during the course of the reading was very small. In roo minutes $\epsilon$ for wave-length 6,080 changed from 0.942 to 0.914 . This amounted to 0.03 per cent. per minute. The values for the fading correction given in Column IX., Table VII., were calculated from this value.

The explosion of the cell prevented the direct determination of the value $\epsilon_{\mathrm{NH}_{3}}-\epsilon_{h}$. To obtain this value the following measurements were made:

(I) The "magnesium" cell filled with water compared with the glass water cell, $\epsilon_{w}-\epsilon_{h}$ (Table III.).

TABLE III.

TABLE V.

Water in Mg Cell-Glass Water Cell.

Water in Na Cell-Glass Water Cell

\begin{tabular}{|c|c|c|c|c|c|c|c|}
\hline$\stackrel{\lambda}{\mu \mu}$ & Cell $R$ & Cell $L$ & $\boldsymbol{\epsilon}_{w}-\epsilon_{h}$ & $\stackrel{\lambda}{\mu \mu}$ & Cell $R$ & Cell $L$ & $\epsilon_{w}^{\prime}-\epsilon$ \\
\hline 664 & 39.53 & 36.54 & .0306 & 664 & 45.72 & 32.83 & \\
\hline 608 & 29.29 & 35.81 & .0358 & 608 & 44.68 & 32.68 & \\
\hline 564 & 39.09 & 35.23 & .0401 & 564 & 45.02 & 33.23 & .131 \\
\hline 529 & & & .0494 & 529 & 45.66 & 32.34 & \\
\hline 502 & 38.41 & 33.70 & .0553 & 502 & 46.09 & 32.16 & \\
\hline
\end{tabular}


(2) The "sodium" cell filled with $\mathrm{NH}_{3}$ compared with the glass water cell, $\epsilon_{\mathrm{NH}_{3}}-\epsilon_{h}$ (Table IV.).

(3) The "sodium" cell filled with water compared with the glass water cell, $\epsilon_{w}^{\prime}-\epsilon_{h}$ (Table V.).

By subtracting (3) from (2) we obtained $\epsilon_{\mathrm{NH}_{3}}-\epsilon_{w}$. Adding to this the value (I), $\epsilon_{w}-\epsilon_{h}$, we obtained the required value $\epsilon_{\mathrm{NH}_{3}}-\epsilon_{h}$ (Table VI.).

TABLE IV.

TABLE VI.

$\mathrm{NH}_{3}$ in $\mathrm{Na}$ Cell-Glass Water Cell. $\quad \mathrm{NH}_{3}$ in $\mathrm{Mg}$ Cell-Glass Water Cell (calc.).

\begin{tabular}{|c|c|c|c|c|c|}
\hline$\stackrel{\lambda}{\mu \mu}$ & Cell $R$ & Cell $L$ & ${ }^{\epsilon} \mathrm{NH}_{3}{ }^{-\epsilon}$ & $\stackrel{\lambda}{\mu \mu}$ & $\epsilon^{\epsilon} \mathrm{NH}_{3}-\epsilon_{h}$ \\
\hline 664 & & & .072 & 664 & -.028 \\
\hline 635 & & & .084 & 608 & +.003 \\
\hline 608 & 43.37 & 33.80 & .098 & 564 & +.021 \\
\hline 585 & & & .107 & 529 & +.034 \\
\hline 564 & 44.03 & 33.15 & .112 & 502 & +.047 \\
\hline 546 & & & .114 & 480 & +.062 \\
\hline 529 & 44.27 & 32.97 & .116 & & \\
\hline 515 & & & .119 & & \\
\hline 502 & 44.35 & 32.44 & .123 & & \\
\hline 491 & & & .128 & & \\
\hline 480 & & & .133 & & \\
\hline
\end{tabular}

TABLE VII.

Magnesium at Room Temperature (about $16^{\circ} \mathrm{C}$.).

\begin{tabular}{|c|c|c|c|c|c|c|c|c|c|c|c|}
\hline \multirow{2}{*}{$\begin{array}{l}\dot{0} \\
z \\
\dot{\alpha} \\
\dot{x} \\
\end{array}$} & \multirow{2}{*}{$\stackrel{\lambda}{\mu \mu}$} & \multicolumn{2}{|c|}{ Reading. } & \multirow{2}{*}{$\begin{array}{l}{ }^{\epsilon} \mathrm{Mg} \\
-\epsilon_{h}\end{array}$} & \multirow{2}{*}{${ }^{\epsilon} \mathrm{NH}_{3}-{ }^{-\epsilon} h$} & \multirow{2}{*}{$\begin{array}{c}{ }^{\epsilon} \mathrm{Mg} \\
-{ }^{\epsilon} \mathrm{NH}_{3}\end{array}$} & \multirow{2}{*}{$\underset{\text { in Mine }}{\text { Time }}$} & \multirow{2}{*}{$\begin{array}{c}\text { Per } \\
\text { Cent } \\
\text { Correc- } \\
\text { tion. }\end{array}$} & \multirow{2}{*}{$\begin{array}{c}{ }^{\epsilon} \mathrm{Mg}-{ }^{\epsilon} \mathrm{NH}_{3} \\
\text { (Cor- } \\
\text { rected). }\end{array}$} & \multirow{2}{*}{$\alpha^{2} / \epsilon$} & \multirow{2}{*}{$\begin{array}{l}\frac{\mathrm{I}}{\lambda^{2}} \times \mathrm{Io}^{-8} \\
\left(\mathrm{Cm} .^{-2}\right) .\end{array}$} \\
\hline & & $a_{3}$ & $a_{4}$ & & & & & & & & \\
\hline 1 & 664 & 80.50 & 4.90 & 1.212 & -.028 & 1.240 & 0 & 0 & 1.241 & 24.0 & 2.26 \\
\hline 2 & 608 & 74.70 & 7.65 & 0.942 & +.003 & 0.940 & 23 & 0.8 & 1.014 & 29.4 & 2.70 \\
\hline 3 & 564 & 70.06 & 10.21 & 0.779 & +.021 & 0.758 & 43 & 1.3 & 0.769 & 38.8 & 3.13 \\
\hline 4 & 529 & 66.90 & 11.18 & 0.705 & +.034 & 0.671 & 57 & 1.7 & 0.684 & 43.6 & 3.54 \\
\hline 5 & 502 & 64.35 & 13.25 & 0.622 & +.047 & 0.575 & 68 & 2.0 & 0.587 & 50.8 & 3.94 \\
\hline 6 & 480 & 62.04 & 13.92 & 0.579 & +.062 & 0.517 & 84 & 2.5 & 0.529 & 56.3 & 4.33 \\
\hline 7 & 608 & 74.87 & 8.51 & & & & 100 & & & & \\
\hline
\end{tabular}

The difference between the extinction coefficients of the blue magnesium solution and of liquid ammonia was then obtained by subtracting the value $\epsilon_{\mathrm{NH}_{3}}-\epsilon_{h}$ from the value $\epsilon_{M g}-\epsilon_{h}$. The measurements made with magnesium are summarized in Table VII., and are plotted in Fig. 2.

\section{SUMmary.}

I. Kraus has shown that the electrical properties of solutions of metals in liquid ammonia can be explained on the assumption that the atoms of 
the metal dissociate into electrons partly combined with the solvent and into the cations present in solutions of its salts in ammonia.

In order to test the assumption that the blue color of these solutions is due to the unsolvated portion of the electrons resulting from this dissociation, the absorption spectra of dilute solutions of sodium and magnesium in liquid ammonia were determined in the visible spectrum.

2. A solution of magnesium and a solution of sodium of the same intensity of color were found to have the same absorption spectrum. This is strong evidence in favor of the assumption that the coloring principle is the same in both cases.

3. Approximate measurements of the concentrations of the sodium solutions make it probable that Beer's law holds for the total dissolved sodium.

4. Qualitatively the evidence is in favor of the assumption that the absorption is due to the unsolvated electrons, but our measurements cannot be interpreted quantitatively in terms of Drude's theory of metallic dispersion.

Chemical laboratory of the University of California,

July I 5 , I9I 5 . 